\title{
General Relativity and the Divergence Problem in Quantum Field Theory
}

\author{
S. DESER* \\ Institute for Theoretical Physics, University of Copenhagen, Copenhagen, Denmark
}

\section{INTRODUCTION}

$\mathrm{P}$ OSSIBLE physical consequences of general relativity for the elementary particle problem have usually been assumed to be negligible, due to the weakness of the gravitational coupling constant. It has been felt that since the effects of the new particles and the energies at which current theory becomes invalid occur very much below the domain for which gravitation becomes significant, a correct future theory will obviate the necessity of considering relativity in this context. It is presumed that, in any case, such a correct theory. will differ so radically from current ideas, that arguments based on these will lose their validity. Further, general relativity is sometimes thought to be purely macroscopic in applicability, and to lose its meaning in subatomic regions where the concept of metric (or possibly metric operator) is not very transparent.

To these classic objections, several levels of reply may be given. Thus, it may be pointed out, for example, that the effect of a theory is not always felt first through its gross direct dynamical contributions. Also, general relativity provides the most natural definition of the stress-energy tensor of matter and, in that sense, underlies any theory dealing with masses and energies of fields and particles. The gravitational field is the only one which by definition is coupled to all matter fields, and always in the same way. The principle of general covariance on which the general theory is based is in no way restricted to macroscopic considerations (elementary particles are affected by gravitational fields), and it is thus necessary to explore its effects on all fields. The fact that an interval or metric component may not be simply measurable "within," say, an elementary particle is no more an argument against the relevance of relativity there than is the complex definition of electron position in an hydrogen atom an argument against the use of coordinates. It appears feasible to extend the usual quantum mechanical measurement theory to cover gravitational couplings also.

The relevance of the general covariance principle to the elementary particle domain has long been recognized by $O$. Klein, from whose program in this connection derive many of the arguments of this section. ${ }^{1}$

Such general arguments may be supplemented with very considerable quantitative ones. The whole scheme of

\footnotetext{
* National Science Foundation Postdoctoral Fellow.

${ }^{1}$ O. Klein, Kosmos 32, 33 (1954); Helv. Phys. Acta Suppl. 4, 58 (1956); and Niels Bohr and the Development of Physics (Pergamon Press, London, 1955), p. 96.
}

current Lorentz-invariant field theory is plagued with divergences occurring because there is no limit to the energies involved in it, while the relevant functions do not decrease rapidly enough with energy to make the integrals exist. No satisfactory cutoff method is known which preserves the basic requirements of physical meaningfulness, and there is now strong doubt as to whether any can be found within a Lorentz-covariant framework. At this point, general relativity may be invoked. Classical considerations indicate that for any kind of matter coupled to the metric field in the Einstein way, there are limitations on the energy densities and masses which can be concentrated or built up in a given region. ${ }^{2}$ In all such investigations, space-time loses its physically meaningful character beyond such limiting densities (or alternately, below such limiting dimensions), singularities then appearing in the solutions. Similarly, a lower bound exists on the size of wave packets built up from linear wave equations, below which gravitational self-effects enter. This bound is independent of the specific field involved; for an energy $E$, and a packet of linear dimension $d, E<c^{4} d / 2 \gamma$, while $E=\hbar c / d$, so that ${ }^{3} d>l_{0}=(2 \hbar c \kappa)^{\frac{1}{2}}$. Thus, below this fundamental length $l_{0} \sim 10^{-32} \mathrm{~cm}$, the packet can no longer be taken to be linear. ${ }^{2}$ Although this distance is very small, it does correspond to a finite energy, and its effects may well be felt earlier. That $l_{0}$ is a universal constant independent of the parameters of particular fields is due to the fact that at these energies the actual masses are negligible, as will be seen below; the adjunction of $\kappa$ to $\hbar$ and $c$ defines a length or energy automatically.

Still more significant is the following. As is well known, under very wide assumptions, theories of coupled fields lead, near the light cone, to singularities in the propagators of the clothed particles and to the existence of at least some infinite renormalization constants, independent of perturbation theory. ${ }^{4}$ All known Lorentzinvariant couplings are of this type, in particular electrodynamics and the renormalizable meson theories.

2 The Schwarzchild solution of the field equations for a perfect liquid, for example, sets limits on the mass of a sphere of given radius and size for given density. General considerations as to requirements on matter to keep nonsingular metrics may be found in A. Lichnérowitz, Théories Relativistes de la Gravitation et de l'Éléctromagnétisme (Masson et Cie, Paris, 1955).

${ }^{3} \gamma$ is the Newtonian, $\kappa$ the Einstein gravitational constant. Henceforth natural units, $\hbar=c=1$ will be used; $\kappa^{\frac{1}{2}}$ has then dimensions of a length.

${ }^{4}$ G. Källén, Physica 19, 850 (1953); H. Lehmann, Nuovo cimento 11, 342 (1954). 
It is just in the basic conditions for the proofs of such "pessimistic" theorems that the gravitational field and its coupling differ profoundly from the matter field interactions. First, it is necessary that there exist an energymomentum vector, $P_{\mu}$, such that the usual commutation relations hold for any operator $0(x)$,

$$
\partial_{\mu} 0(x)=i\left[0, P_{\mu}\right] \text {. }
$$

This is not the case in the general theory, and is very significant there; a $P_{\mu}$ can only be defined for a closed system in such a way as to be independent of the choice of inner coordinates, ${ }^{5}$ that is, in terms of over-all properties of the system, and therefore cannot yield values (or derivatives) of quantities which are connected with inner properties or regions. The nonlinearity, and consequent lack of simple translational properties, of parts of the system by themselves are here brought out; attempts to fix precisely the position of an arbitrary component of a "clothed" electron, for example, would clash with the energy density bounds mentioned earlier. Further, matter and gravitational energies are not invariantly separable.

A second, related, departure from usual theory lies in the form of the interaction terms. Contrary to the assumption always tacitly made there, the matter Lagrangian simply has no free-particle part any longer; gravitational coupling enters in the kinetic energy, in a multiplicative fashion, and one cannot really disentangle a noninteracting particle here. The equal-time matter commutation relations also depend on the position in space-time involved (and therefore on the matter distribution itself) by way of the now dynamical metric tensor. ${ }^{6}$ The differences due to this fundamentally different mode of coupling will occur most critically at high energies, and forbid any approximations from "free-particle" just at the point where difficulties now exist. In particular, then, matter fields will no longer be representable by linear wave equations in the high-energy domain. ${ }^{7}$ The free motion varies with the geometry of the space involved, which is in turn conditioned by the matter and its motion. It will be seen in Sec. III that the results inferred in this investigation as to the nonsingularity of propagators are based in good part on just these characteristics of gravitational coupling. It may well be that a more adequate future theory of particles will incorporate some of these aspects of the nonlinear gravitational interaction. Certainly, if it be a field theory, it must escape the pessimistic conclusions for current theory by some such channel. If so, it may be all the more

${ }^{5}$ W. Pauli, Encyklopädie der Mathematischen Wissenschaften (B. G. Teubner, Leipzig, 1921), Vol. V2, pp. 539-775.

${ }^{6}$ For a given nonflat geometry, the situation would essentially be the same as the flat space-time one. That is, by suitable modification, the content of Eq. (1) could still be maintained.

${ }^{7}$ It might be thought that by writing $g_{\mu \nu}=\delta_{\mu \nu}+0(\kappa)$ such a free part would be obtained. However such expansions in $\kappa$ are invalid at high energies. The limiting process as $\kappa$ approaches zero will itself be seen to proceed in a more subtle way than such an expansion of gravitational quantities in powers of $\kappa$. instructive to see how an improvement seems to emerge here; in any case, the gravitational cutoff may remain as a backstop to such possible convergent future theories.

\section{QUANTIZATION}

Once gravitational interaction is introduced in the microscopic domain, it is necessary that the corresponding field be quantized; from the fact that the stresstensor is an operator, it follows that the curvature tensor must be one as well. ${ }^{8}$ While it is conceivable that a classical approximation for the metric quantities may suffice to secure convergence of field theory (through the above mentioned classical consideration), it is important to verify that the full quantized theory is also finite. It is outside the scope of this paper to discuss the quantization problem and the difficulties to which it may give rise when applied to the gravitational field. The method to be employed here to effect the quantization is that of Feynman. ${ }^{9}$ It is known to be equivalent to the canonical one for the usual (quadratic Lagrangian) fields, and seems to avoid at least some of the difficulties encountered by extensions of the canonical method to this highly nonlinear problem. Whether or not the two procedures differ somewhat in this case, the Feynman method appears to provide a consistent approach. The question of which components are dynamically independent and which fully determined by the matter quantities need not, in a certain sense, be known beforehand. ${ }^{10}$ This prescription has the further advantage, for our purposes, of exhibiting explicitly the crucial differences, stressed earlier, between matter and gravitational interactions. The general framework is as in ordinary theory: the vacuum expectation value of the operator of interest is the functional integral (suitably weighted and normalized) of a corresponding $c$-number quantity over all classical configurations of the system. It may be expected, roughly, that the contributions of the "scalar" and "transverse" parts of the gravitational field, though not really separable, will be to limit the density of matter in a given region for the former, and to "smear out the light cone" for the latter. This phrase refers to the expectation that the summation over all possible Riemann spaces corresponding to the various classical possibilities will average away the uniqueness of the Minkowski light cone, and with it the singularities of the propagators on such a cone. Such an effect would

${ }^{8}$ While such "self-consistent" approaches as starting from $R_{\mu \nu}=-\kappa\left\langle T_{\mu \nu}\right\rangle$ may be considered, they are only approximate in nature.

${ }^{9}$ For a recent discussion of the functional integral procedure, see P. T. Matthews and A. Salam, Nuovo cimento 2, 120 (1955).

10 Some of the possible difficulties of Feynman quantization of general relativity have lately been resolved: C. Misner, Revs. Modern Phys. 29, 497 (1957), discusses the proper choice of integration variables; B. Laurent, Nuovo cimento 10, 1445 (1956), considers the invariance of the integrals under general coordinate transformations. Laurent also has clarified other aspects of the choice of variables and of the quantization problem (private communication). 
realize a possibility also remarked on by Pauli at the Bern relativity conference in $1955^{11}$

The usual field theory is known to be fully determined by knowledge of all vacuum propagators, that is, vacuum expectation values of all powers of the various field operators. From these, the various $S$-matrix elements are directly obtainable. In our case, the strong nonlinearity makes the relations between propagators and physical quantities more complicated (so that their physical significance is less immediate); however, they still determine the theory, and their singularities, if any, are still the relevant ones.

\section{MATTER PROPAGATORS}

We are interested in comparing the one-particle Green's function $G^{\prime}$, corrected for both electromagnetic (or mesic) and gravitational self-effects, with $S_{F}^{\prime}$, the flat space electromagnetically corrected one. The latter behaves at least as singularly on the light cone as the free propagator, $S_{F}(p)=(\gamma \rho+m)^{-1}$; this is sufficiently singular to yield infinite constants. It is shown below that near the cone, $G^{\prime}$ reduces to $S_{F}^{\prime \prime}$, the propagator corrected for gravitation only. Therefore it is sufficient to show that $S_{F}^{\prime \prime}$ is less singular than $S_{F}$. The complete problem, as represented by $G^{\prime}$, can then be expected to remain nonsingular, the omitted terms not being relevant in this connection. Alternately, the use of electrons and photons clothed by gravitation as the noninteracting elements of electrodynamics seems a sufficiently good approximation to bring out the finiteness brought into the problem by gravitation. Manyparticle Green's functions are expected to display similar features at high energies, with the possible additional display of a bias against too close approach between the various particles (also to be expected on classical grounds). The self-effects constitute the main problem in any case. Gravitational field propagators can also be constructed, and should also behave nonsingularly; Schwarzschild-type singularities ought not to appear, since matter is now "regularly" distributed, while the "transverse" propagators should also be well behaved (since the gravitational self-interaction acts in the same way as it does on matter). We restrict ourselves to matter Green's functions here.

There should not appear any gravitational coupling renormalization, as the mass $m$ plays the dual role of charge and (inertial) mass and the two types of renormalization, if any, must be equal by the equivalence of these two masses. Since there is only one type of "charge" here, no vacuum polarization is expected; the mass renormalization should also vanish in view of the absolute definition of mass and energy in the general theory, and of the fact that the observed mass is automatically defined from the coupling term between gravitation and matter, and through this by the value of $P_{0}$ for the system.

${ }^{11}$ W. Pauli, Helv. Phys. Acta Suppl. 4, 69 (1956).
We first examine the usual Lorentz-covariant theory, to bring out the inevitable singular behavior of the Green's function of any field on the light cone, regardless of coupling. As a concrete example, we consider neutral scalar meson theory. Here the one-nucleon propagator, $S_{F^{\prime}}{ }^{\prime}$, is given by ${ }^{9}$

$$
\begin{aligned}
S_{F}^{\prime}\left(x-x^{\prime}\right) & \equiv i\left\langle 0\left|\left(\psi(x) \bar{\psi}\left(x^{\prime}\right)\right)_{+}\right| 0\right\rangle \epsilon\left(x, x^{\prime}\right) /\langle 0 \mid 0\rangle \\
& =N^{-1} \int \psi(x) \bar{\psi}\left(x^{\prime}\right) \exp (i A[\psi, \varphi]) \delta \varphi \delta \psi \delta \bar{\psi}
\end{aligned}
$$

where the action $A$ is

$$
\begin{array}{r}
A=\int \bar{\psi}(x)\left(-i \gamma_{\mu} \partial_{\mu}+m\right) \psi(x)+g \int \bar{\psi}(x) \psi(x) \phi(x) \\
+\int \phi(x)\left(-\square^{2}+\mu^{2}\right) \phi(x)
\end{array}
$$

and the normalizing factor $N$ corresponds to the vacuum-vacuum transition amplitude,

$$
N=\int \exp (i A) \delta \varphi \delta \psi \delta \delta \bar{\psi}
$$

$\epsilon$ is a sign factor, and the " + " sign a chronological ordering operator corresponding to outgoing wave boundary conditions. Integration over the nucleon variables can be performed, giving

$$
\begin{array}{r}
S_{F}^{\prime}\left(x-x^{\prime}\right)=N^{-1} \int G\left(x, x^{\prime} ; \varphi\right) \exp \left[i A_{M}+A_{0}\right] \delta \varphi \\
{\left[-i \gamma_{\mu} \partial_{\mu}+m+g \varphi(x)\right] G\left(x, x^{\prime} ; \varphi\right)=\delta\left(x-x^{\prime}\right),}
\end{array}
$$

where $A_{M}$ is the free meson field part of $A, A_{0}$ an additional contribution due to closed loop effects; this term is a functional of $\varphi$ but does not depend on the coordinates explicitly. The problem is thus resolved into two parts : obtaining the solution of Eq. (6) for $G$, and performing the integral in Eq. (5) with this result for $G$. The rigorous solution for $G$ may be written ${ }^{12}$

$$
G\left(x, x^{\prime} ; \varphi\right)=S_{F}^{(0)}\left(x-x^{\prime}\right) F_{1}\left(x, x^{\prime} ; \varphi\right)+F_{2}\left(x x^{\prime} ; \varphi\right) .
$$

$F_{1}$ is a function everywhere regular in $x, x^{\prime}$ and approaching unity at the origin, $x=x^{\prime} . S_{F}{ }^{(0)}$ is the simple uncoupled Dirac propagator $S_{F}$ with mass equal zero. ${ }^{13}$ $F_{2}$ is holomorphic everywhere but on the light cone, $\left(x-x^{\prime}\right)^{2}=0$ where it may display a logarithmic singu-

${ }^{12}$ The analysis used here of solutions of (6), (11), and the more general equation (14) was developed by J. Hadamard, Lectures on Cauchy's Problem (Dover Publications, New York, 1952). His work was improved by M. Riesz, Acta Math. 81, 1 (1949). The method of Riesz was adapted to the Dirac equation by H. Malmheden, Comm. Lund 8, 1 (1947). Since the relevant results are the same for our purposes, we deal mostly with the second-order equation for simplicity.

${ }^{13}$ The mass $m$ can also be reinstated in $S_{F}$, the difference being absorbed in the definition of $F$. 
larity; in some cases $F_{2}$ is null. The functional dependence on $\varphi$ enters only in the $F_{i}$; further, we neglect $F_{2}$ here, since we are interested in the leading singularity on the light cone, and $S_{F}$ is worse than logarithmic there.

Upon inserting (7) for $G$ in the functional integral, we see that the singular $S_{F}\left(x-x^{\prime}\right)$ can be taken outside the integral. The integral of $F$, as $\left(x-x^{\prime}\right)^{2} \rightarrow 0$ may either converge absolutely in which case it represents a constant coefficient, the so-called $Z_{2}$ renormalization, on the cone, or, if it is illegitimate to interchange this limit with the integration, $G^{\prime}$ has an even worse singularity than $S_{F}$ on the cone. At the origin $G^{\prime}$ is just equivalent to $S_{F}{ }^{(0)}$, since $F_{1}=1$ there. ${ }^{14}$ This theorem, proved earlier by other methods, ${ }^{4}$ applies to a wide class of couplings, including all usual field theories. It expresses the fact that, as the arguments of any field's Green's function approach the light cone, the effects of its couplings can never result in smoother behavior than the free particle one, and probably cause a more singular variation.

In the parallel problem of interaction of a matter field (chosen here as scalar for simplicity) with the gravitational one, the total action is

$$
A=\int(-g)^{\frac{1}{2}}\left[x^{-1} R+\frac{1}{2}\left\{-\phi \square^{2} \phi+\mu^{2} \phi^{2}\right\}\right]\left(d^{4} x\right),
$$

where

$$
\square^{2} \equiv(-g)^{\frac{1}{2}} \partial_{\mu}\left(g^{\mu \nu}(-g)^{\frac{1}{2}} \partial_{\nu}\right),
$$

where $R$ is the scalar curvature. Interaction between the two fields arises through the metric dependence of the matter part of the action. Again we wish to compute

$\Delta_{F}^{\prime \prime}\left(x, x^{\prime}\right) \equiv i\left\langle 0\left|\left(\phi(x) \phi\left(x^{\prime}\right)\right)_{+}\right| 0\right\rangle /\langle 0 \mid 0\rangle$

$$
=N^{-1} \int \phi(x) \phi\left(x^{\prime}\right) \exp (i A)(\delta g) \delta \phi,
$$

where

$$
N=\int \exp (i A)(\delta g) \delta \phi .
$$

The functional integration includes a summation over all possible Riemannian spaces preserving the signature $(+++-)$, the $(\delta g)$ representing the appropriate variables with respect to which the integral is taken. We do not need their precise form; they might, for example, be the product of the 16 Vierbein variables at each point of space-time. ${ }^{10}$ The arguments $x, x^{\prime}$ of $\Delta^{\prime \prime}$ may at this stage be thought of as two quadruples of numbers representing two arbitrary points in a fourdimensional manifold, the integration over all possible Riemann spaces being an average of the two-point

\footnotetext{
${ }^{14}$ The singularity of present field theory may be restated by the fact that in principle, at $x=x^{\prime}, G^{\prime}=S_{F}$, but that the limit $x \rightarrow x^{\prime}$ yields an infinite proportionality constant between the two functions.
}

function over all possible ways of imposing a metric structure on this manifold, consonant with the signature and possibly certain topological requirements. Eliminating the matter variables by performing the $\varphi$ integration (which corresponds to the $\psi$ integration in the previous case), we get

$$
\begin{gathered}
\Delta_{F^{\prime}}{ }^{\prime \prime}\left(x, x^{\prime}\right)=N^{-1} \int \Delta\left(x, x^{\prime} ; g_{\mu \nu}\right) \\
\times \exp \left(i A_{g}+i A_{0 g}\right)(\delta g) \\
\left(-\square^{2}+\mu^{2}\right) \Delta\left(x, x^{\prime} ; g\right)=\delta\left(x, x^{\prime}\right)
\end{gathered}
$$

where, in analogy to the previous case, $A_{g}$ is the pure gravitational action, $A_{0 g}$ an addition to it due to the $\varphi$ integral. Once again we must insert into (10) the rigorous solution $\Delta$ of (11). Here,

$$
\Delta\left(x, x^{\prime} ; g\right)=s_{g}^{-2}\left(x, x^{\prime}\right) F_{1}\left(x, x^{\prime} ; g\right)+F_{2}\left(x, x^{\prime} ; g\right) \text {. }
$$

$s_{g}{ }^{-2}$ is the inverse square of the geodesic distance between the two points $x$ and $x^{\prime}$ in the space represented by the metric $g_{\mu \nu}(x)$ (there is a geodesic between two sufficiently close points in any Riemann space), $F_{1}$ is again a regular function everywhere, equal to unity at the origin, while $F_{2}$ has a logarithmic singularity on the light cone in the space (that is along all null geodesics issuing from $x^{\prime}$ ) if $\mu \neq 0$, and is again not of interest. The crucial difference between our previous examples and gravitation appears here: while the leading factor is, in strict analogy with the earlier problem, just the inverse square of the interval between $x$ and $x^{\prime}$, it depends now on the dynamical variables of the metric field, and therefore cannot be removed outside the functional integral. There is no free particle propagation, since this factor also interacts with the metric. This is obviously true whatever the matter field is; all are coupled to $g_{\mu \nu}$. Had we considered $G^{\prime}$, that is, included other interactions as well, we would have obtained at this stage for the totally corrected propagator $G^{\prime},{ }^{15}$

$$
\begin{aligned}
G^{\prime}\left(x, x^{\prime}\right)=N^{-1} \int(\delta g) \exp \left(i A_{g}+i A_{0 g}\right) \\
\quad \times \int(\delta \chi) \exp [i A(\chi)] G\left(x, x^{\prime} ; \chi, g\right),
\end{aligned}
$$

where $\chi$ stands for all these additional interactions (electromagnetic, mesic, etc.) and

$$
\left(-\square^{2}+g_{\mu}[\chi] \partial_{\mu}+h[\chi]\right) G\left(x, x^{\prime} ; \chi, g\right)=\delta\left(x x^{\prime}\right) .
$$

The $\chi$ dependence enters only through the $g_{\mu}$ and $h$, the coefficients of the second derivatives being the same as in (11), that is, only gravitational quantities. The

${ }^{15}$ The two-particle propagator would have another $G\left(y, y^{\prime} ; g, \chi\right)$ in the integrand, the meson-nucleon one $\chi(\xi) \chi\left(\xi^{\prime}\right)$ there: in either case there appear to be no new singularity problems. It might even be expected that the distance between two-particles must exceed $l_{0}$. 
general solution of (14), however, has its singularities determined entirely by the second derivative structure. That is why $s^{-2}\left(x, x^{\prime}\right)$ is always the leading term, and in usual theory can be moved out of the $\chi$ integral, but not past the gravitational integration. Incidentally, the equivalence of $\Delta^{\prime \prime}$ and $G^{\prime}$ for purposes of investigating the effect of gravitation on the singular behavior is thereby established; at high enough energies all usual couplings become unimportant compared to the gravitational. Returning therefore to our result, (12), for $\Delta\left(x, x^{\prime} ; g\right)$, we must now perform the functional integral, that is average over all spaces, with weight $\exp i\left(A_{g}+A_{0 g}\right)$ and measure $(\delta g)$. We may again neglect $F_{1}$ and concentrate on $s^{-2}$, which has the same sort of singularity on the cone of the Riemann space that $\left(x-x^{\prime}\right)^{-2}$ has in Euclidian space. The average of this quantity, $\left\langle s^{-2}\right\rangle$, will give the leading behavior for all fields, since the details of other couplings, as well as masses, etc. are absorbed into the $F^{\prime}$ s. Thus, if a cutoff appears (and $l_{0}$ is the only length now left) it will endow all particles with about the same "structure." Evaluation of the integral is not explicitly feasible. However, the following qualitative argument is in favor of its being nonsingular. Consider the two points $x$ and $x^{\prime}$ to lie on each other's light cones in some given Riemann space, say, flat space. Then there exist, for any two such points, "almost" no other spaces for which this is simultaneously also true. "Almost" none means here that very stringent integrodifferential equations must be satisfied by the metrics of such other spaces. Further, the singularities of $s^{-2}$ as a functional of the metric variables are not very strong. In analogy to the usual theory of integration, any measure in the function space which gives meaning to $\mathcal{S}(\delta g)$ will be such as to have no contributions from these "isolated" spaces. They will have measure zero. Conversely, if the two points are not joined by a null geodesic in flat space, there will be "almost" no other spaces in which they are so connected, and no new singularities will be brought in. The weight factor of the gravitational action, having magnitude unity, can only help in rendering the integration finite; it will also act (as the corresponding factor does in normal theory) to oscillate away the contributions from spaces very far away from the classical solutions, which make the phase large in a random way. A rigorous proof of our conjectures would seem to require a better developed mathematical theory of measure in functional integrations. Certain over-all properties of $G^{\prime}$ (or $\Delta^{\prime \prime}$ ) can be obtained from corresponding ones of $\Delta$; in particular, under interchange of $x$ and $x^{\prime}$ and of boundary conditions, these Green's functions remain invariant; they are not, however, functions of $\left(x-x^{\prime}\right)$ since the spaces involved are not isotropic and homogeneous, unlike the flat one.

If we wish to consider effects near the light cone due to one of the ordinary interactions, whose contributions vanish at the cone, as we have seen, we may try to expand in powers of the interaction in question. For example, in the case of a scalar particle coupled to another, we have

$$
\begin{gathered}
G^{\prime}=N^{-1} \int(\delta g) \exp \left(i A_{g}\right) \\
\quad \times \int \delta \varphi \exp \left(i A_{\varphi}\right) \Delta\left(x, x^{\prime} ; \varphi, g\right) \\
\left(-\square^{2}+\mu^{2}+g \varphi\right) \Delta=\delta\left(x, x^{\prime}\right) .
\end{gathered}
$$

Then we expand the $F_{i}$ entering in the solution for $\Delta$, and particularly $F_{1}$, since it comes in with the $s^{-2}$ dominant term. Thus,

$$
\begin{aligned}
& \Delta \sim \mathcal{S}_{g}{ }^{-2}\left(x, x^{\prime}\right)\left[\left.F_{1}\left(x, x^{\prime} ; \varphi\right)\right|_{\varphi=0}+g^{2} \int(d y)\right. \\
& \left.\quad \times(d z) \varphi(y) \varphi(z) \delta^{2} F_{1} /\left.\delta \varphi(y) \delta \varphi(z)\right|_{\varphi=0}+\cdots\right] .
\end{aligned}
$$

The $\varphi$ integration can be performed, and $\varphi(y) \varphi(z) \rightarrow$ $\Delta(y, z ; g)$. It may be thought that the infinities of perturbation theory (or indeed of local field theory in general, since its infinities first appear at this stage) will still arise in the integration over the intermediate points, because in any Riemann space (of the sum) overlapping singularities on the light cone still occur. However, the integration over the gravitational variables is to be performed first in order to include their averaging effect, before the intermediate integrals are done. Only then would the expansion be expected to make sense, since these apparent new singularities will have been smoothed out by the same argument as that given for $\left\langle s_{g}^{-2}\right\rangle$ above. Equivalently, the fluctuations of the matter field at a point may no longer be infinite, so that ${ }^{16}$

$$
\lim _{x \rightarrow x^{\prime}}\left\langle\varphi(x) \varphi\left(x^{\prime}\right)\right\rangle=\lim \left\langle s_{g}^{-2}\left(x, x^{\prime}\right)\right\rangle<\infty .
$$

In principle, the renormalization constants of, say, electrodynamics, are now presumably rendered finite by general relativity and can therefore be evaluated.

${ }^{16} \mathrm{~J}$. Schwinger (unpublished lecture notes, Stanford 1956) relates the inconsistency of local field theory with the infinity arising at this stage, due to $\left\langle\varphi(x) \varphi\left(x^{\prime}\right)\right\rangle \rightarrow \infty$ as $x \rightarrow x^{\prime}$. The gravitationally corrected theory is radically different from the usual one, so that care must be taken to use the now proper forms of such quantities as the physical mass or the equal time matter commutators (which now depend on the metric). As Schwinger has pointed out, the inconsistency of the usual theory results from the fact that it permits arbitrarily small space-time measurements, which, however, turn out to yield inconsistent (infinite) results because the interaction effects are always felt, however short the interval employed; while the equal time commutators require that no interaction effects be felt for sufficiently small times. When gravitation is taken into account, such arbitrarily short measurements cannot be made, as they would clash with the bounds established by general relativity; thus, the vacuum fluctuations at a point or the overlap of light-cone singularities of two-fields may no longer contribute to make results infinite for physical quantities. 
The main effects of relativity, in fact, being high energy in nature, will enter into these constants, which absorb the asymptotic contributions in the theory. Asymptotic expansions of the functions of the theory could also be made, if everything were convergent, as this type of cutoff would be, by its very definition, physically acceptable. It is likely that the correct relation between the bare and physical charge and the cutoff depends strongly on the bare charge, which now, however, would be a finite parameter. ${ }^{17}$ Of course, one could not attempt such expansions by transcribing directly into flat space-time language the form of the propagators obtained from general relativity, as they would then seem to violate some probability conservation or other physical requirements, since the very concept of cutoff in flat space-time violates them. Unfortunately, even the generally covariant form of the finite propagators seems difficult enough to extract from the functional integrals. ${ }^{18}$

The flat space theory is expected to emerge in the limit of vanishing gravitational coupling constant; however, since the latter appears in the total action as $\kappa^{-1} A_{g}$, this limit is quite different from the usual adiabatic shutting off procedure. In fact, $\kappa \rightarrow 0$ is equivalent to $\hbar \rightarrow 0$ in this term, that is, the classical limit; as is well known, the latter corresponds to having the phase stationary, in this case, $\delta A_{g}=0$, i.e. the empty space field equations

$$
R_{\mu \nu}=0 .
$$

These equations become equivalent to the conditions for Euclidian space-time since we are dealing with closed systems, and asymptotically flat metrics accordingly. The $\mathcal{S}(\delta g)$ then acts to replace the metric wherever it occurs in the matter action or in the integrands by its Minkowski values. This decoupling procedure brings out the significance of the background flat space-time which serves as basis for classical measurements on the system. The flat space is thus analogous to the "in" and "out" quantities defined by means of the corresponding adiabatic decoupling in usual field theory, but in addition, the gravitational decoupling turns the metric quantities into $c$ numbers.

\section{CONCLUSIONS}

We have seen that a qualitative estimate of the gravitationally corrected matter propagators does

${ }^{17}$ L. D. Landau [Niels Bohr and the Development of Physics (Pergamon Press, London, 1955), p. 52] discusses an approximation to usual field theory which would imply a breakdown of the latter below a cut-off distance of about $l_{0}$.

18 Practical evaluation of such functional integrals as Eq. (10) is beset by the difficulties that any tractable expansion loses some vital features of the full theory, such as nonlinearity, general covariance, self-interaction, etc. Thus the linear approximation to the gravitational field corresponds to reinstatement of the privileged character of the Minkowski metric, since gravitation now propagates like any linear field in flat space-time. Further, this approximation on the metric entering in the matter part is probably invalid just at the high energies of interest. It is hoped to treat some of the approximation methods in a future publication. indicate that singularities corresponding to point particles and interactions disappear. A corroborative argument, mentioned in Sec. I, comes from consideration of the energy-momentum vector, $P_{\mu}$, and its commutation relations. In a flat space-time, and effectively (by suitable extension) in a nonflat but externally given geometry, Eq. (1) holds. $P_{\mu}$ determines the translation properties of the system, and because of the homogeneity and isotropy of the space, of any part of the system as well. In a space-time which interacts with matter, however, this relation ceases to be purely kinematical, and in fact no longer holds. $P_{\mu}$ does have, as a canonical conjugate, the center-of-mass coordinate, $X_{\mu}$, of the system with respect to a flat background frame. However, $P_{\mu}$ cannot be used to locate closely any component of the system. The proof that any component field, if focused upon sufficiently, behaves as if uncoupled, which forms the basis of the difficulties in Lorentz-invariant theories, here becomes invalid, since it requires a $P_{\mu}$ with all the usual properties. It may be argued that the propagators themselves lose some of their meaning by the same argument, since they deal with localized effects of fields. This is true only to the extent that physical quantities always involve space-time integrals of such functions. The nonsingularity of the $\Delta^{\prime \prime}$ and $G^{\prime}$ is an expression of the fact that one can never force such concentration of energy of a field that its gravitational couplings cease to matter-the induced geometry would cause a repulsion before such a stage could be reached.

Thus, a quantum field theory including gravitational effects seems to rid itself of point-singularity difficulties which neither part alone could avoid; classical relativity did tolerate point singularities in its solutions, which this more complete framework has no place for. Nonsingular matter distributions and nonsingular metric go hand in hand. This can be ascribed partly to the intimate coupling of geometry and kinetic energy, leading to limitations on energy concentrations, partly to the indefiniteness of the line element: the "smearing of the light cone" due to the appearance on an equal footing with Euclidian space of all other suitable Riemann spaces.

Even if the infinities are thus removed, the physical problems of obtaining correct dynamical descriptions of the interactions of observed particles (to say nothing of explaining their existence) are left untouched at this stage of the theory. However, it seems comforting that inclusion of the total, unmutilated general covariance avoids the formal difficulties to be found in Lorentz-invariant theory, and it may be that in the question of the values of the observed parameters, $l_{0}$ will play a significant role.

Since within the framework of flat space-time one could consider theories with interactions built into the kinetic energy terms, it is an interesting question whether finiteness can be achieved merely by such an 
extension, or whether the free parts of those fields which play such a role need, in addition, to be nonlinear in some specific way, or whether only general relativistic coupling will work. Equation (1) would still hold for such extended theories, being a purely kinematical property of homogeneous space-time, but the usual local commutativity properties would be lost; that is, the commutator of a field component with its time-derivative would, even at equal times, depend on other fields. Alternately, the conjugate momentum would be a function of such other fields as well as of the time derivative of the corresponding component. The assumption that this is not the case appears to be implicitly required in the proofs of the pessimistic theorems. The commutation relations are closely related to the creation and annihilation of particles; if they now depend on the other fields present, it might happen that the contributions, as the thresholds of higher and higher creation processes are passed with increasing energies, are damped thereby. These contributions from new creation processes seem to be the cause (or another expression) of the divergences in field theory, in which cases the new couplings might yield convergence. Such couplings arise, for example, in quantum hydrodynamics. ${ }^{19}$ Saturation might be expected to occur with some of these couplings; that is, the presence of many quanta, or high energies, may damp further creation. ${ }^{20}$

\section{ACKNOWLEDGMENTS}

I am most thankful to Professor O. Klein for many valuable discussions of this work; his contributions to all its phases have been extremely illuminating.

The author also wishes to acknowledge the hospitality accorded him at the Institute for Theoretical Physics. His work was performed during tenure of a National Science Foundation Fellowship.

${ }^{19}$ A. Thellung, Helv. Phys. Acta 29, 103 (1956). In this case the kinetic energy has the form $\rho v^{2} / 2$, both $\rho$ and $v$ being fields. This form may be very different, however, from one in which several variables $\rho_{\mathrm{i} j}$ multiply $v_{\mathrm{i}} v_{j}$.

${ }^{20}$ Landau $^{17}$ has also remarked that at high energies field theory might go over into a quantum hydrodynamical scheme.

\title{
Negative Mass in General Relativity
}

\author{
H. BONDI \\ King's College, University of London, London, England
}

1.

\begin{abstract}
$A^{1}$ LTHOUGH some of the arguments relating to negative mass are fairly elementary and well known, it will nevertheless be of advantage to rediscuss the meaning of this term. In the first instance, without fully specifying a theory, we can distinguish between three kinds of mass according to the measurement by which it is defined: inertial, passive gravitational, and active gravitational mass. Inertial mass is the quantity that enters (and is defined by) Newton's second law*; passive gravitational mass is the mass on which the gravitational fields acts, that is it is defined by $\mathbf{F}=-m$ $\operatorname{grad} V$; active gravitational mass is the mass that is the source of gravitational fields and is hence the mass that enters Poisson's equation and Gauss' law.

In Newtonian physics the law of action and reaction implies the equality of active and passive gravitational masses, but the equality of inertial mass with these other two is a separate empirical fact. The sign of both these masses can take either value and it is an additional empirical result that it is always positive. Four cases accordingly arise, if this empirical fact is left out of account.
\end{abstract}

\footnotetext{
* A mass-independent force (say, of electromagnetic nature) has to be used here, for obvious reasons.
}

(i) All mass is positive; this is familiar.

(ii) Inertial mass negative, gravitational mass is positive. A body consisting of matter of this kind will respond perversely to all forces whether gravitational or of other kinds, but will produce gravitational forces just as a usual body does.

(iii) Inertial mass positive, gravitational masses negative. In this case we would have normal behavior relating to all nongravitational forces, but gravitational behavior involving masses of this type and of type (i) would be governed by a negative Coulomb law; i.e., like masses would attract and unlike masses would repel.

(iv) All mass is negative. This would be a combination of (ii) and (iii). Matter of this kind responds perversely to nongravitational forces, responds like ordinary matter to gravitational forces, but produces repulsive gravitational fields.

In general relativity the situation is quite different. The principle of equivalence is not a separate fact but is basic to the theory. Accordingly the ratio of inertial and passive gravitational masses is the same for all bodies. The relation between active and passive gravitational masses is not fixed by anything like Newton's third law as this would require integrals over 